\title{
Reawakening anaesthesia research
}

\author{
Anaesthesia - one of the greatest achievements of medicine_remains unexplained, but a slew of new \\ studies may help to solve the mystery
}

\author{
Andrea Rinaldi
}

$\mathrm{E}$ ach year, more than 230 million surgical procedures are performed under general anaesthesia worldwide, and most of us will experience going under at some point in our lives. We take that dreamless sleep through an otherwise unbearably painful procedure for granted, yet it is one of the greatest achievements of medicine. "Before October 1846, surgery and pain were synonymous. These gruesome interventions were carried out with requisite haste and inevitable commotion. Absent was the precision and calm that we associate with modern surgery. It consisted of pulling, cutting, sewing, sawing, manipulation of joints, and slicing of skin", wrote Daniel Robertson and Alexander Toledo in their recent account of the invention of anaesthesia [1]. Although attempts at putting patients to sleep had been reported since ancient times, the dawn of modern anaesthesia is unanimously set on 16 October 1846, when surgeon John Collins Warren removed a tumour from the neck of a local printer with the help of a young Boston dentist, William T.G. Morton, who had discovered that inhaled ether made tooth extraction painless (Fig 1).

But oddly enough, despite the fact that general anaesthesia has been around for some 170 years and that anaesthetics are among the most common drugs administered to humans, our knowledge of how they work has not advanced much since Morton's times. No generally accepted mechanism has so far been proposed that might explain how a host of inhaled or injected compounds can induce a reversible state characterized by unconsciousness, amnesia, analgesia and a lack of movement, while our physiological system remains stable. It is a fascinating scientific challenge, and the field of general anaesthesia is experiencing a great revival, with a variety of studies on both the molecular effects of anaesthetics and the changes they induce at the level of neural circuits and systems.

\section{"Before October 1846, surgery and pain were synonymous. These gruesome interventions were carried out with requisite haste and inevitable commotion."}

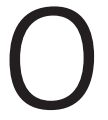

ne of the complications of anaesthesia is the bewildering variety of molecules in use, which range from the monoatomic noble gas xenon to the 56atom steroid alfaxalone, through the phenol propofol and the halogenated compounds halothane and isoflurane. Since they found it impossible to establish a clear structureactivity relationship, early researchers turned their attention to the physicochemical properties of anaesthetics, noticing that they were soluble in organic solvents rather than in water. In 1899 and 1901, respectively, Hans Horst Meyer, a German pharmacologist and Director of the Pharmacological Institute at the University of Marburg, and Charles Ernest Overton, a British cell biologist and pharmacologist, independently published evidence correlating a substance's ability to dissolve in lipids and its anaesthetic properties. The so-called Meyer-Overton theory of anaesthesia postulated that once an anaesthetic agent reaches a critical concentration in neuron lipid membranes, it interferes with brain functioning and causes narcosis.
Revised by subsequent observations, the lipid theory has remained the leading hypothesis of general anaesthesia for most of the $20^{\text {th }}$ century. However, this has not impeded the exploration of alternative routes. In 1961, Linus Pauling came out with his own original theory, attributing the action of anaesthetic agents to "the formation in the brain of minute hydrate crystals of the clathrate type" [2]. Notwithstanding Pauling's eminence in the scientific community of the time, however, his theory remained largely ignored-also because it was never proved experimentally-and "now stands as little more than a footnote in the history of anaesthesiology" (http:// paulingblog.wordpress.com/category/theoryof-anesthesia/).

\section{"One of the remarkable} features of general anaesthesia is that it acts on animals as evolutionarily distant as Caenorhabditis and humans."

As time went by, evidence accumulated that proteins rather than lipids could be the targets of general anaesthetics. In 1984, Nicholas Franks and William Lieb showed that anaesthetics inhibited an enzyme, firefly luciferase, at anaesthetising concentrations, suggesting that, despite their chemical and structural diversity, general anaesthetics might act by competing with endogenous ligands for specific receptors [3]. Given that general anaesthesia is a complex phenomenon involving amnesia, unconsciousness, analgesia and immobility, with each component mediated by different 


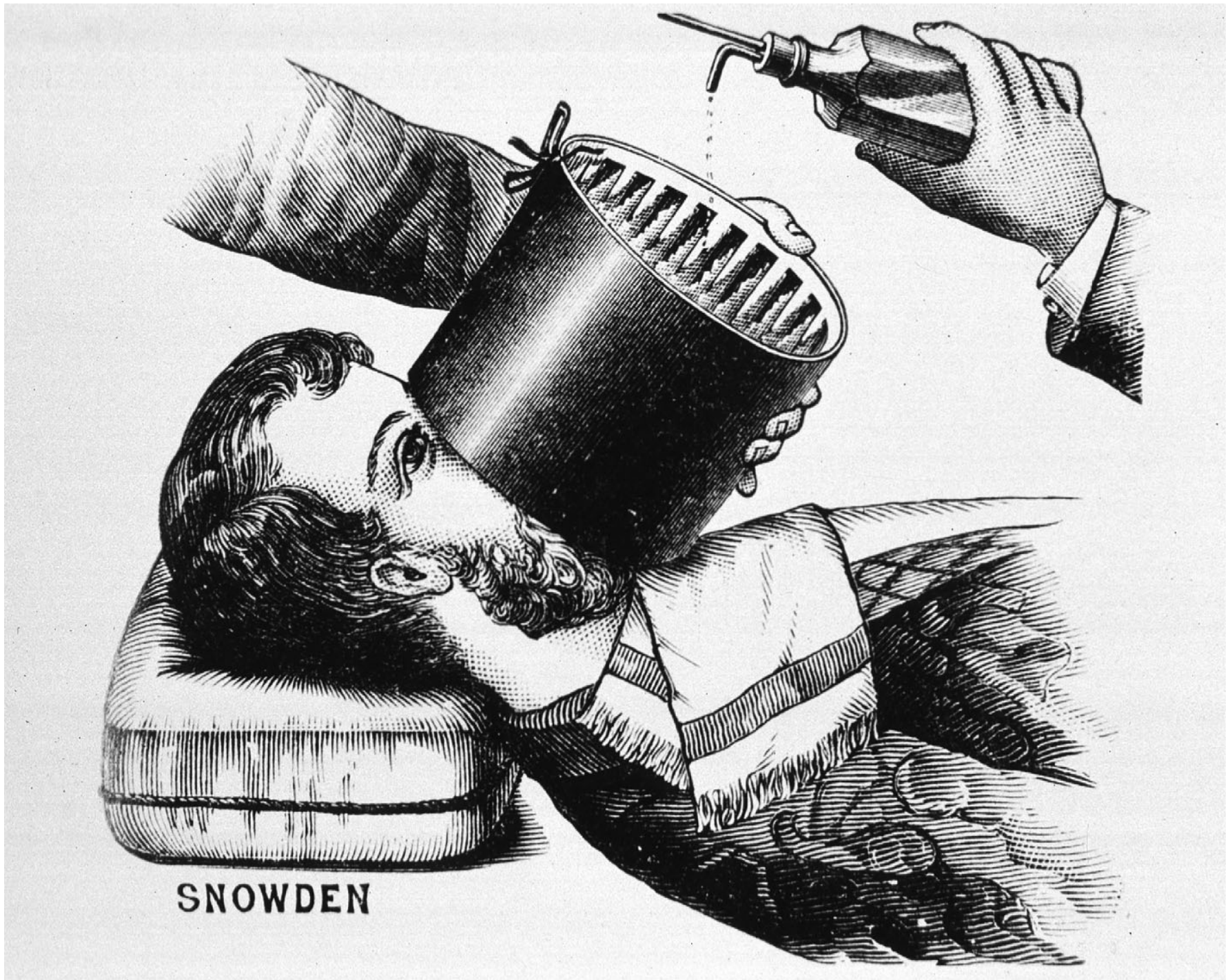

Figure 1. Anaesthesia inhalation, the old way.

Allis' Ether Inhaler: a circular apparatus, slotted to accept a weave of fabric and fitted with an outer covering, is placed over a reclining patient's nose and mouth; ether is being administered from a bottle, a few drops at a time onto the fabric. Allis' instrument can be considered as a development of the original Morton's inhaler (see main text). Wood engraving, published in Artificial anaesthesia. A manual of anaesthetic agents and their employment in the treatment of disease, by Laurence Turnbull, Blakiston, Philadelphia, 1896. Credit: US National Library of Medicine, History of Medicine Division.

receptors and neuronal circuits, it is logical to expect interactions between anaesthetics and a plethora of potential target proteins, each of which can in principle be both activated or de-activated by the drug. Of all the possible targets, neurotransmitter-gated and voltage-gated ion channels have emerged as the most likely candidate targets for general anaesthetics. In particular, the role of $\gamma$-aminobutyric acid type A $\left(\mathrm{GABA}_{\mathrm{A}}\right)$ receptors is becoming increasingly clearer [3]several general anaesthetics are known to enhance the action of GABA on receptors or to reduce the desensitization of receptors- while the effects on voltage-gated ion channels are still largely unknown.

"Given that the activity of voltage-gated ion sodium channels has been shown to be significantly affected by anaesthetics at physiological concentrations, several questions remain to be answered", said Vincenzo Carnevale, from Temple University in Philadelphia (PA, USA). "Are these channels always inhibited or, perhaps in some cases, also activated? Do all general anaesthetics share the same set of binding sites and mechanism of action? Are voltage-gated sodium channels sensitive to all general anaesthetics or only to a subset of them?" Carnevale and his team have addressed these questions using "flooding" molecular dynamics simulations [4,5]. This type of simulation assumes that drug molecules are present in large excess with respect to the other components (sodium channel, lipids, water and ions, in this case), describing a situation in which an extremely large concentration of anaesthetic has been administered. These drug molecules are monitored for long time intervals as they engage in either temporary or stable interactions with a protein; the large number 
A

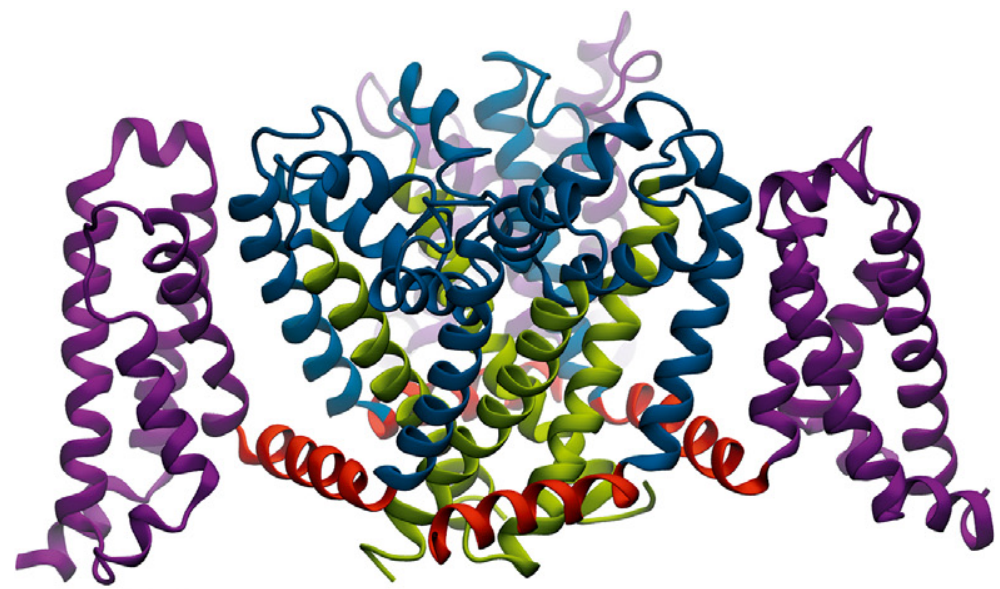

B

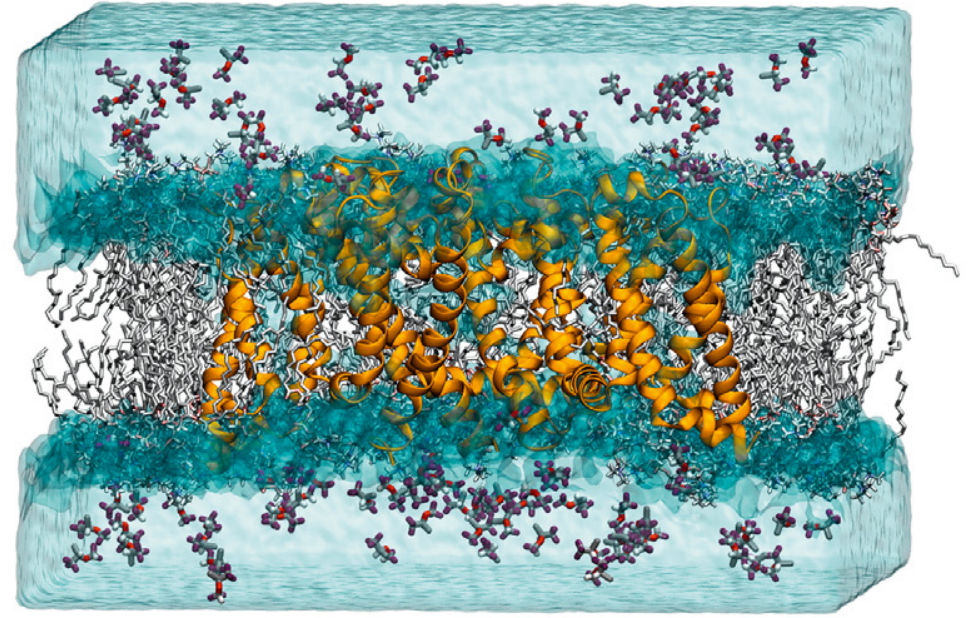

C

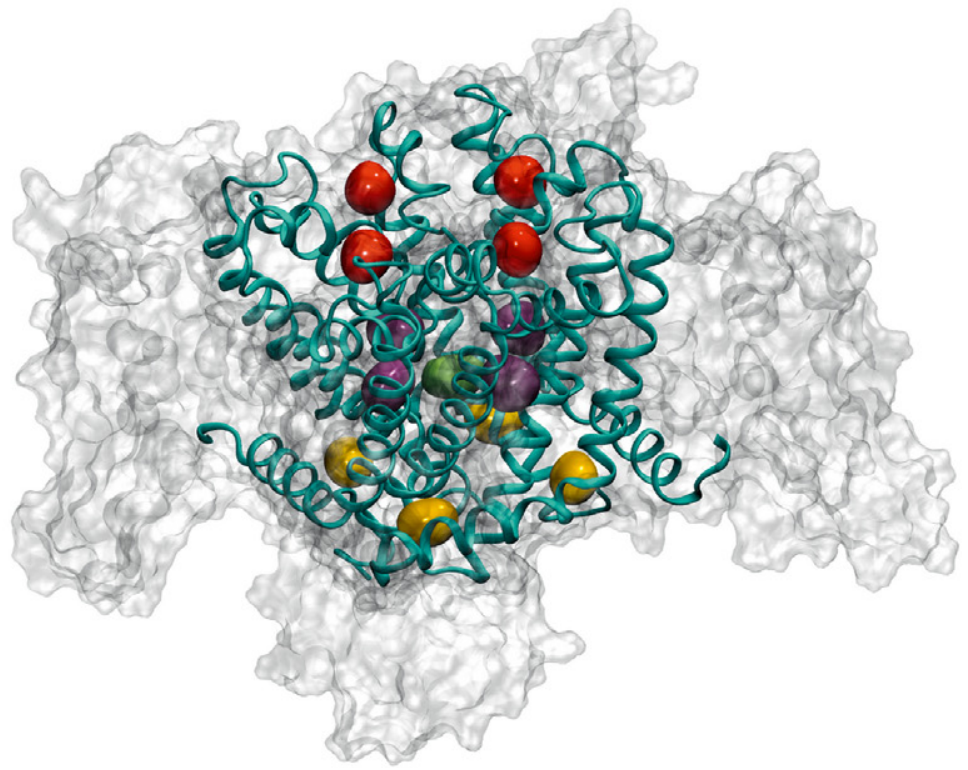

Figure 2. Molecular dynamics simulation of isoflurane binding sites in the archetypal bacterial voltage-gated channel NaChBac.

(A) Structural domains of $\mathrm{NaChBac}$ showing the voltage-sensing domain (purple), connected through the $\mathrm{S4}-\mathrm{S5}$ linkers (red) to the pore domain (blue/green) with the $\mathbf{S 6}$ helices highlighted in green. (B) Isoflurane flooding simulation system initial set-up showing the NaChBac protein (orange) in the lipid bilayer (white) with isoflurane molecules in the aqueous phase. (C) The three binding sites identified by clustering analysis: extracellular site (red), linker site (yellow) and cavity site (purple/green). From [4]. Reproduced with permission. of drug molecules gives researchers confidence that all the potential interaction sites on the surface of the protein will be eventually explored. Carnevale explained that the result of this "blind-search" is the set of regions of the protein surface that are potential binding sites for anaesthetics (Fig 2).

"The 'flooding' approach allowed us to discover the binding sites of the inhaled anaesthetics isoflurane and sevoflurane on a bacterial sodium channel without any prior knowledge of the binding process", Carnevale said, adding that the most surprising finding has been the fact that several drug molecules are able to bind the channel simultaneously at different locations. "This observation has potentially deep consequences, as it implies that a different modulation of the channel activity should be expected depending on which binding site is occupied", he said. "Since the pattern of occupancy is dependent on the concentration of drug molecules, modulation of sodium channels by general anaesthetics shows a complex dependence on the amount of anaesthetics present in solution. Accordingly, we found experimentally that sevoflurane favours activation at low concentrations and additionally accelerates current decay at high concentrations."

$\mathrm{F}$ ollowing this trail, although moving in a different direction, a team led by David Worcester at the University of California, Irvine, USA, and Michael Weinrich at the Eunice Kennedy Shriver National Institute for Child Health and Human Development in Bethesda, MD, USA, is exploring whether general anaesthetics might work by affecting the order of lipids surrounding membrane-embedded ion channels and thus the function of the channels themselves. "Many proteins important for cell signalling, both pre- and post-synaptic, appear to localize in lipid raft domains and there is increasing evidence that the lipid environment can modulate their functions", Worcester explained.

The researchers used lamellar neutron and X-ray diffraction to show that the conventional inhalational anaesthetics halothane and xenon change the phase distribution of lipid raft mixtures at physiological concentrations to favour the disordered phase $[6,7]$. As shifts in lipid phase distribution can produce substantial modifications of the mechanical properties of biological membranes, thus influencing the functioning of ion channels 
Sidebar A: The quest for novel anaesthetic drugs

General anaesthetics are indispensable tools, but not devoid of side effects. Propofol for instance, one of the most commonly used anaesthetics, can lower blood pressure and produce respiratory depression, particularly in the elderly. As clinical practice has to meet the needs of an increasingly older population, the demand for both safer and better anaesthetics - easier to administer and with rapid onset of action and recovery - has increased constantly in recent years. "All of the general anaesthetic agents currently in clinical use were first developed more than 30 years ago. This lack of progress has been due, in large part, to our poor understanding of how these drugs act to produce their therapeutic actions and their undesirable side effects. But that's changing, and researchers are using recently acquired knowledge to design better drugs", said Douglas Raines from the Massachusetts General Hospital in Boston, MA, USA.

One approach is to modify the molecular structures of existing anaesthetic agents with the goal of improving their pharmacology. This rational design strategy has been used with etomidate as the molecular template and produced remimazolam, a promising analogue of the commonly used benzodiazepine-type sedative midazolam that induces fast onset of sedation and quick recovery [13]. "The other approach is to use high-throughput screening to identify completely novel molecular templates upon which to base new anaesthetic agents", said Raines. "Although this latter approach requires designing new assays and may be higher risk, it has the potential to lead to the development of completely new classes of anaesthetics with unique and valuable properties." Following this approach, Rod Eckenhoff and his group at the University of Pennsylvania in Philadelphia (PA, USA) recently screened a library of more than 350,000 compounds, identifying a probe compound representing a single novel chemotype, currently under study [13].

in which they are located, these observations reinforce the idea that anaesthetics might exert their effects, including probably side effects, through mechanisms that do not involve direct interactions with proteins. Intriguingly, when tested under similar conditions, a non-anaesthetic compound (1,2-dichlorohexafluorocyclobutane) with chemical properties similar to halothane did not alter the domain structure of lipid model membranes [6,7]. "Anaesthesia must ultimately result from a change in ion currents through the protein pores of excitable

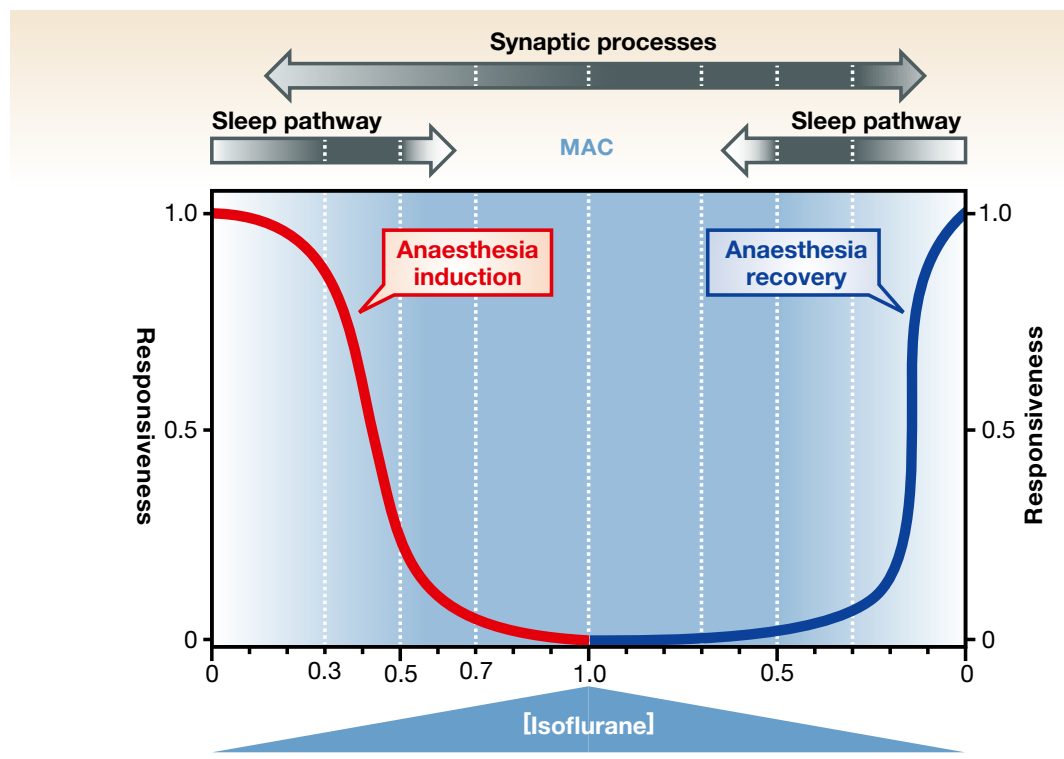

Figure 3. General anaesthesia model.

Induction of isoflurane anaesthesia (red curve) might involve potentiation of an endogenous sleep pathway first, at lower drug concentrations ("Sleep pathway" arrow, darkness intensity represents maximal anaesthetic effect, e.g. 0.3-0.5 vol\% isoflurane), followed by non-specific attenuation of synaptic neurotransmission across the entire brain, at surgical (e.g. 0.7-1.0 vol\% isoflurane) concentrations ("Synaptic processes" arrow, darkness intensity represents maximal anaesthetic effect). Recovery from general anaesthesia (blue curve) would first require recovery of synaptic coordination across the brain prior to awakening. These distinct effects on synaptic processes and sleep pathways could explain the inertia that is typically observed during recovery from general anaesthesia. MAC, minimum alveolar concentration required for performing surgery. Adapted from [11].

Reproduced with permission.

membranes in the nervous system. However, volatile anaesthetics are rather unselectivethey affect the function of many ion channelsand their potencies are tightly correlated with their solubilities in lipids", Weinrich explained. "Our demonstration that volatile anaesthetics shift the equilibrium for phase mixing in lipid raft mixtures provides a plausible mechanism whereby these agents can change the lipid environment surrounding ion channels and thus modulate their function."

A nother fresh and potentially revolutionary contribution to solving the mystery of general anaesthesia molecular mechanisms comes from a group led by Luca Turin at the Alexander Fleming Biomedical Sciences Research Centre in Vari, Greece. Turin's group has shown that volatile general anaesthetics such as xenon cause a rapid and reversible increase in the electron spin content of Drosophila and that these electron spin changes differed substantially in anaesthesia-resistant mutants [8]. One of the remarkable features of general anaesthesia is that it acts on animals as evolutionarily distant as Caenorhabditis and humans. Turin and colleagues were also able to calculate that xenon and other volatile anaesthetics perturb the electronic structure of a protein model. "Our results establish a possible link between [un]consciousness in flies and electron currents, detectable by a change in a quantum observable, electron spin", they concluded [8].

While xenon is usually believed to act by inhibiting N-methyl-D-aspartate (NMDA) receptors non-competitively, with little effect on $\mathrm{GABA}_{\mathrm{A}}$ receptors or non-NMDA glutamatergic receptors, the results of Turin and co-authors open up a completely new scenario. "Suppose, then, that there exists, in one or more proteins essential to central nervous system function, a hydrophobic site lined with an electron donor on one side and an acceptor on the other side. When general anaesthetics enter the site, they could connect donor to acceptor by creating a pathway for electron transfer where there was no pathway", the authors wrote [8]. This hypothesis is consistent also with the Meyer-Overton relationship, in the sense that anaesthetics would target hydrophobic pockets within proteins. "We have proposed several possibilities that could account for a connection between electrons and 


\section{Sidebar B: Mastering anaesthesia in laboratory mammals}

An important but often neglected aspect of anaesthesia is its impact on experimental outcomes in biomedical research that uses vertebrates. Researchers involved in imaging studies seem to be particularly aware of the problem. "Imaging in animals requires anaesthesia for obvious welfare and practical reasons, to restrain gross motion and to induce unconsciousness to minimize stress response", said veterinarian Jordi Lopez-Tremoleda at Queen Mary University of London, UK "Anaesthetic agents have a profound effect on the physiology of the animal and thereby will impact on their systems/organ/tissue homeostasis." The most important and critical adverse effects are depression of respiration, cardiovascular system disruption and thermoregulation [14] Thus, to avoid confounding results and a lack of reproducibility, it is important to ensure that appropriate protocols are in place during preclinical imaging, including appropriate animal handling, choice of anaesthetic regimes, the use of physiological monitoring, and to consider factors such as the humidity and temperature of the laboratory, and its impact on the animal's body temperature, Lopez-Tremoleda remarked. "Many of these may seem trivial to many of us when we are busy running extensive high-throughput studies but the truth is that they may have confounding effects on the image data acquired", he said.

"Anaesthesia should be tailored to the circumstances, not just the continuation of what has 'worked for the past 20 years"', confirmed Brenda Klaunberg from the NIH Mouse Imaging Facility in Bethesda (MD, USA), adding that further research is needed to define the physiological side effects of anaesthetic agents that might impact on preclinical models. "We are really only beginning to understand some of the subtle strain differences, especially in mice, that may alter their response to both different anaesthetics and any test treatment", said Anthony Nicholson, from the University of Adelaide in Roseworthy, South Australia. "This aspect is likely to have even greater impact as research continues to identify more specific aspects of the mechanisms of anaesthesia, receptors directly and indirectly affected and the associated effects peripheral to anaesthesia." "However, protocols for animal preparation for anaesthesia are generally not detailed on these issues. Similarly, literature on the impact of repeated anaesthesia remains scarce", continued Lopez-Tremoleda. Most imaging devices have very limited physiological monitoring systems, and most of the systems available are manufactured by very few companies. Apparently not a very profitable area for investment, developments on monitoring systems are very limited. "Companies would require some support to commit to new developments in the field but so far that has not been strategically supported", Lopez-Tremoleda noted.

anaesthesia, but it's too early to identify a single, general mechanism linking perturbations of electron currents in a model organism, as we observed, to central nervous system physiology. We are just at the beginning of a long, fascinating journey”, Turin commented.

\section{"The only thing we are sure about consciousness, is that it is soluble in chloroform."}

$\mathrm{T}$ he effects of anaesthetics must ultimately be related to molecular interactions, but studies aimed at explaining general anaesthesia at the level of neuronal circuits are also thriving. A recent, detailed electrophysiological study showed that anaesthesia and sleep do share more than apparent similarities. Max Kelz and colleagues at the University of Pennsylvania in Philadelphia, USA, demonstrated that isoflurane directly causes sleep-promoting neurons deep within the hypothalamus to fire, suggesting that general anaesthetics could lead to unconsciousness by "hijacking" sleep circuits in the brain [9].

However, this might not be the entire story. Starting from the evidence that although all animals can be rendered unresponsive by similar concentrations of anaesthetics, not all animals sleep, Bruno van Swinderen and Benjamin Kottler at the University of Queensland, Brisbane, Australia, have proposed a theory that tries to reconcile the "sleep-centric" view of general anaesthesia, with observations that unconsciousness derives from attenuation of neurotransmission, followed by fragmentation of neuronal networks and loss of coherence across either the brain [10] or simpler nervous systems [11]. "We propose that general anaesthesia may be effectively described as a two-step process involving first the activation of endogenous sleep pathways, followed by a global attenuation of synaptic release across the brain", the authors wrote (Fig 3).

Noticing that in non-sleeping systems, like adult nematodes, sensitivity to anaesthetics can be altered by modifying a single pre-synaptic protein, syntaxin $1 \mathrm{~A}$, van Swinderen and Kottler identified the membrane-bound SNARE complex, a key component of the synaptic release machinery in all animals, as an important target of general anaesthetics [11]. An intriguing aspect of their hypothesis is that it brings together the observation that anaesthetics interact with specific proteins such as $\mathrm{GABA}_{\mathrm{A}}$ receptors with older hypotheses focused on the lipid bilayer of neurons. For instance, isoflurane and propofol, which act on $\mathrm{GABA}_{\mathrm{A}}$ receptors, promote sleep at relatively low doses but compromise neurotransmission at higher doses, possibly by concentrating in neuron membranes and interfering with synaptic vesicle fusion and thus neurotransmission.

cc

". . our knowledge of disorders of consciousness, such as coma and persistent vegetative state, could well benefit from anaesthesia studies."

"The only thing we are sure about consciousness, is that it is soluble in chloroform”, said Turin. Given that the definition itself of consciousness is elusive, and given that it is even more baffling how it arises in our brains, many believe that research into how anaesthesia operates might lead to a better understanding of how the brain sees itself and the world around it. In addition, our knowledge of disorders of consciousness, such as coma and persistent vegetative state, could well benefit from anaesthesia studies. Scientists, using brain-imaging techniques, electroencephalography, and other approaches, have tried to spot a discrete region of the brain that could link the action of anaesthetics to loss of consciousness. Many brain areas seem to be associated with anaesthetic effects, especially a posterior lateral corticothalamic complex centred around the inferior parietal lobe [12]. Anaesthetics would then produce unconsciousness either by blocking the interactions among specialized brain regions or by shrinking the number of activity patterns available to cortical networks [12]. Most likely though, the mystery of anaesthesia is not going to be solved anytime soon. Difficult as it might be, however, revived interest and numerous well-focused studies 
are bringing us closer to consciousness over unconsciousness.

\section{References}

1. Robinson DH, Toledo AH (2012) Historical development of modern anesthesia. J Invest Surg 25: 141-149

2. Pauling $L$ (1961) A molecular theory of general anesthesia. Science 134: 15-21

3. Franks NP (2014) The unfolding story of how general anaesthetics act. In: The Wondrous Story of Anesthesia, Eger II El, Saidman LJ, Westhorpe RN (eds), pp 597-608. New York: Springer

4. Raju SG, Barber AF, LeBard DN, Klein ML, Carnevale V (2013) Exploring volatile general anesthetic binding to a closed membrane-bound bacterial voltage-gated sodium channel via computation. PLoS Comput Biol 9: e1003090

5. Barber AF, Carnevale V, Klein ML, Eckenhoff RG, Covarrubias M (2014) Modulation of a voltage-gated $\mathrm{Na}^{+}$channel by sevoflurane involves multiple sites and distinct mecha-

nisms. Proc Natl Acad Sci USA 111:

$6726-6731$

6. Weinrich $\mathrm{M}$, Nanda $\mathrm{H}$, Worcester DL, Majkrzak CF, Maranville BB, Bezrukov SM (2012) Halothane changes the domain structure of a binary lipid membrane. Langmuir 28: $4723-4728$

7. Weinrich M, Worcester DL (2013) Xenon and other volatile anaesthetics change domain structure in model lipid raft membranes. J Phys Chem B 117: 16141-16147

8. Turin L, Skoulakis EMC, Horsfield AP (2014) Electron spin changes during general anesthesia in Drosophila. Proc Natl Acad Sci USA 111: E3524-E3533

9. Moore JT, Chen J, Han B, Meng QC, Veasey SC, Beck SG, Kelz MB (2012) Direct activation of sleep-promoting VLPO neurons by volatile anaesthetics contributes to anesthetic hypnosis. Curr Biol 22: 2008-2016
10. Lewis LD, Weiner VS, Mukamel EA, Donoghue JA, Eskandar EN, Madsen JR, Anderson WS, Hochberg LR, Cash SS, Brown EN et al (2012) Rapid fragmentation of neuronal networks at the onset of propofol-induced unconsciousness. Proc Natl Acad Sci USA 109: E3377-E3386

11. van Swinderen B, Kottler B (2014) Explaining general anesthesia: a two-step hypothesis linking sleep circuits and the synaptic release machinery. BioEssays 36: 372-381

12. Alkire MT, Hudetz AG, Tononi G (2008) Consciousness and anesthesia. Science 322: $876-880$

13. Chitilian HV, Eckenhoff RG, Raines DE (2013) Anesthetic drug development: novel drugs and new approaches. Surg Neurol Int 4(Suppl 1): $\mathrm{S} 2-\mathrm{S} 10$

14. Tremoleda JL, Kerton A, Gsell W (2012) Anaesthesia and physiological monitoring during in vivo imaging of laboratory rodents: considerations on experimental outcomes and animal welfare. EJNMMI Res 2: 44 